\title{
Habilitación profesional. \\ Condiciones para el aseguramiento de la calidad de la educación médica y condiciones para la confianza recíproca. Experiencia y visión de ASOFAMECH
}

\author{
Octavio Enríquez $L^{1}$, Beltrán Mena $C^{2}$ \\ The actions of the Association \\ of Faculties of Medicine in Chile \\ (ASOFAMECH) to assure the quality \\ of medical education in Chile
}

The aim of ASOFAMECH is to assure the quality of medical education. It promoted a development plan that in 1999 put into motion the accreditation of careers, that soon should be mandatory. Since December 2003, the National Medical Examination is applied and graduates from Medical Schools take it voluntarily. Since 1979, ASOFAMECH created an accreditation system for different training programs for specialists. In 2003 the incorporation of these programs to the Law of Quality Assurance of Superior Education was requested. In 1984, ASOFAMECH participated in the creation of the National Corporation to Assess and Certify Medical Specialties (CONACEM). Recently, the Health Superintendence was created. This organism will gather a national registry of specialists. ASOFAMECH, Scientific Societies, The Chilean College of Physicians and the Academy of Medicine proposed that a title obtained from an accredited training program in a University or a certificate emanated from CONACEM should be requisites for the incorporation to this registry. These are the actions taken by ASOFAMECH to assure the quality of medical education in Chile (Rev Méd Chile 2005; 133: 483-94).

(Key Words: Certification; Education, medical, graduate; Schools, medical; Societies, medical; Specialty boards)

Recibido el 14 de enero de 2005. Aceptado el 21 de enero de 2005.

${ }^{1}$ Presidente de la Asociación de Facultades de Medicina de Chile (ASOFAMECH).

${ }^{2}$ Director de Examen Médico Nacional.

Es ste artículo presenta la visión de la Asociación de Facultades de Medicina de Chile (ASOFA$\mathrm{MECH}$ ) sobre las condiciones para el ejercicio de la Medicina en nuestro país, refiriéndolo especial- mente al actual escenario de la globalización del comercio, con la consiguiente movilidad internacional de profesionales, y la necesidad de definir cuáles son las condiciones para la confianza 
recíproca en este proceso. Se trata entonces, de precisar cuáles son los mecanismos necesarios para asegurar la calidad del ejercicio profesional. Médicos que den seguridad y confianza a los usuarios de los servicios.

Dentro de los objetivos fundacionales de ASOFAMECH, y que han motivado en forma permanente su quehacer a través del tiempo, están el aseguramiento de la calidad de la Educación Médica de pre y postgrado y su vinculación con las políticas y programas de salud del país.

En la década 1990-99, se configuró en Chile un nuevo escenario para la Educación Médica caracterizada por:

1. Un perfil epidemiológico de transición, en que las enfermedades crónicas no transmisibles y el trauma se constituyen en objetivos prioritarios de salud. La coexistencia de dos subsistemas -el público y el privado- cada uno con sus debilidades propias y la insatisfacción de los usuarios, a pesar de los buenos índices de salud del país y la creciente percepción de la necesidad de una profunda reforma del sector orientada a la equidad, calidad y eficiencia de la atención en salud.

2. El vertiginoso ritmo de desarrollo y renovación del conocimiento y la tecnología, con la consiguiente progresiva especialización en la práctica médica.

3. Un marco legal en educación superior que estimula el rápido crecimiento de la oferta de carreras de medicina, con muy pocos y débiles sistemas de control de calidad. Esto trae como consecuencia, además, una perturbación creciente de la tradicional y productiva relación docente-asistencial entre el sistema público y las universidades tradicionales, agrupadas en ASOFAMECH, ya que muchos Servicios de Salud ven en las ofertas de nuevas Escuelas de Medicina una oportunidad para allegar fondos a los debilitados presupuestos hospitalarios. Junto a lo anterior, se produce un gran aumento en la inmigración de médicos extranjeros, la mayoría de ellos de acuerdo a convenios que no exigen ninguna evaluación de las competencias del profesional.

4. En lo ético, el cambio de la relación médicopaciente paternalista por una más horizontal, en el marco de la institucionalización de los derechos de los pacientes. A ello se agrega el mayor nivel de información general de la población sobre la temática médica y una creciente exigencia de calidad de las prestaciones sanitarias por parte de la población usuaria.

5. Bajo la legalidad vigente, un chileno en posesión de su título de médico cirujano, tiene el derecho de ejercer libremente la profesión de por vida. El título, a su vez, puede ser otorgado por cualquiera universidad chilena con reconocimiento oficial. Todas las escuelas de medicina en Chile, han establecido un programa de formación de siete años de duración. Durante los cinco primeros, el alumno adquiere los conocimientos y destrezas en el ámbito de las ciencias básicas, biológicas y sociales y en el de la patología y clínica que le permiten acceder al grado de Licenciado en Medicina. Para obtener el título profesional de médico cirujano, se requiere cumplir un programa de práctica profesional supervisada de dos años, en las principales áreas clínicas de la medicina y la aprobación de los respectivos exámenes finales de carácter teórico y práctico.

Los requisitos exigidos a un médico extranjero para ejercer en Chile, dependen de la existencia o no de convenios entre Chile y su país de origen:

a) Médicos provenientes de países sin convenio. Estos deben someterse a un examen conocido como Examen de Reválida, que el estado ha delegado en la Universidad de Chile. Este examen cubre aspectos teóricos y prácticos y se aprueba superando un puntaje mínimo.

b) Médicos provenientes de países con convenio de reconocimiento automático de título. Estos países son Colombia, Ecuador, Uruguay y Paraguay. Estos médicos sólo deben presentar su título de médico en el Ministerio de Relaciones Exteriores de Chile para certificar su validez, trámite que una vez cumplido les permite ejercer indefinidamente.

c) Médicos provenientes de países con tratados especiales, poseen requisitos distintos. Las actuales negociaciones con el Mercosur pueden cambiar la situación, ya que, de aprobarse los acuerdos (Memorándum de Entendimiento de Ministros de Educación Mercosur - 1998), cualquier egresado de una Escuela de Medicina perteneciente a una universidad acreditada Mercosur podrá ejercer libremente en otro país 
miembro, cumpliendo el criterio de trato nacional. Las negociaciones de libre comercio con la Comunidad Europea y otros países, pueden modificar aún más el escenario.

Para enfrentar los desafíos que el nuevo escenario descrito plantea, ASOFAMECH hizo un análisis estratégico entre los años 1995 y 1997, con numerosos seminarios a lo largo del país, que culminó con la formulación de un plan nacional de desarrollo, para implementarlo en los años venideros. Dentro de las principales propuestas de este Plan de Desarrollo de ASOFAMECH, se contempló:

Propuestas del Plan:

- Reformulación del perfil del egresado y reforma curricular.

- Profesionalización de la función docente.

- Creación de un sistema de evaluación del proceso enseñanza-aprendizaje (Acreditación).

- Creación de un sistema de evaluación del producto (Examen Médico Nacional).

- Fortalecimiento del proceso de acreditación de programas y centros formadores de especialistas de postítulo.

- Perfeccionamiento y potenciamiento de la certificación de especialistas médicos por CONACEM.

- Cuidado y desarrollo de la relación docenteasistencial.

En lo que a desafíos curriculares se refiere, la casi totalidad de las Facultades de Medicina, se encuentran implementando procesos de cambio consistentes con el nuevo escenario descrito. Igualmente los departamentos u oficinas de Educación Médica en cada una de nuestras Facultades, han impulsado programas de capacitación y de investigación educacional, para consolidar la profesionalización de la función docente en las diferentes universidades.

En lo que a acreditación de carreras de Medicina se refiere, el proceso que condujo a la implementación del sistema, pionero en el país, se fue desarrollando en las siguientes etapas:

Acreditación de carreras de Medicina:

- Se formulan los estándares de calidad de la acreditación (1996-1997).
- Propuesta de ASOFAMECH al Consejo Superior de Educación y formación del Comité Técnico.

- Se trabajan y sancionan los estándares.

- Convenio ASOFAMECH-CSE (1998) para iniciar procesos de acreditación.

- Creación de la Comisión Nacional de Acreditación de Pregrado (CNAP) (1999).

- Se conviene con ASOFAMECH que la CNAP se haga cargo del proceso.

- Se crea el Comité de área de Medicina de la CNAP.

- Se sancionan los estándares.

Entre agosto de 2000 y marzo de 2002, se completó el proceso de acreditación de seis carreras de Medicina de ASOFAMECH. Tres de ellas, fueron acreditadas por el período máximo de siete años y otras tres, por un período de dos.

En lo que a Convenios Docentes-Asistenciales y asignación a campos clínicos se refiere, ASOFAME$\mathrm{CH}$, después de un largo proceso y trabajo a nivel de la Comisión Nacional Docente Asistencial, logró convenir con el Ministerio de Salud (MINSAL) una norma general administrativa, el año 2001, que establece pautas, principios y criterios que los Servicios de Salud deben aplicar en los Convenios Docentes-Asistenciales. En lo principal, se busca la estabilidad a largo plazo de los convenios y se establece que no debe existir cohabitación de dos escuelas de medicina en un mismo campo clínico. A pesar de esta normativa del Ministerio de Salud, en varios Servicios de Salud ella no ha sido respetada y se siguen presentando conflictos de cohabitación forzada, lo que ha sido representado por ASOFAMECH a la autoridad sanitaria.

En lo que a la formación de postítulo se refiere, ASOFAMECH completó el séptimo ciclo de acreditación de programas y centros formadores de especialistas que se desarrolló entre 2001 y 2003, el que culminó en septiembre de 2003 con un seminario en que participaron representantes de MINSAL, MINEDUC, Colegio Médico, Academia de Medicina, Sociedades Científicas y las diferentes Escuelas de Medicina, en el que se conoció el informe final de la Comisión Nacional de Acreditación de ASOFAMECH.

La certificación de especialistas en las diferentes disciplinas médicas, a cargo de la Corporación Nacional Autónoma de Certificación de Especialidades Médicas (CONACEM) desde 1984, en la 
cual participa ASOFAMECH junto a representantes de Sociedades Científicas, Colegio Médico y Ministerio de Salud, ha permitido reconocer a más de 8.000 médicos especialistas, los que han debido acreditar sus competencias por medio de un título de un programa universitario acreditado por ASOFAMECH 0 a través de un examen teórico y práctico ante una comisión de especialistas de CONACEM, exigiéndoseles una experiencia previa de cinco años en un servicio clínico calificado.

En lo que a aseguramiento de la calidad de la formación médica de pregrado se refiere, ASOFAMECH inició, con gran esfuerzo y en forma pionera en el país, la acreditación de las carreras bajo la conducción de la Comisión Nacional de Pregrado (CNAP). Sin embargo, hay que tener presente que la acreditación asegura la calidad adecuada del proceso de formación, es decir, garantiza que una escuela reúne las condiciones de formar médicos de buen nivel, pero nada dice de cada uno de los profesionales que de ella egresan. La única forma de conseguir esto último, es sometiendo a cada médico a una evaluación personal. Ambas actividades, acreditación, que evalúa proceso formador, y examen individual del producto terminal de la carrera, que son los egresados, son complementarias y deben coexistir para beneficio de las escuelas, de los estudiantes y de la población.

\section{EXAMEN MÉDICO NACIONAL}

Las Facultades de Medicina agrupadas en ASOFA$\mathrm{MECH}$, acordaron, en 1999, el estudio e implementación de un examen final común a ser rendido por todos su egresados de la carrera de medicina. A este examen se le conoce como Examen Médico Nacional (EMN). Los objetivos del examen se detallan más adelante y uno de su primeros usos, será exigir su rendición como requisito en la asignación de cupos para los programas de especialización otorgados por las escuelas pertenecientes a ASOFAMECH.

Para el estudio de la idea, se formó una comisión compuesta por dos representantes de cada Escuela. Esta comisión sesionó en varias oportunidades y escuchó las opiniones de las Escuelas de Medicina que no pertenecen a ASOFAMECH, así como las de representantes del
Colegio Médico y los Ministerios de Salud y Educación. Consideró además, la experiencia española y estadounidense en un seminario realizado en octubre de 2000, en que expusieron la Dra. Emilia Sánchez (comisión MIR) y el Dr. Robert Galbraith (NBME) (Examen a Médicos Internos Residentes y National Board of Medical Examiners, respectivamente). A este seminario se hizo una invitación amplia que incluyó otras Escuelas de Medicina e instituciones.

El trabajo de esa comisión concluyó con la entrega, en octubre de 2000, de un informe en que se establecía el marco para la implementación de un EMN.

ASOFAMECH aprobó el informe y decidió la elaboración de un proyecto detallado para su implementación. Para este fin, se creó una nueva comisión, con sólo un representante por escuela, conocida como Comisión 2001. Dicha comisión trabajó en varios grupos, concretando los conceptos del primer informe y recabando información práctica a través de la recolección de preguntas, simulación de trabajo de comités e implementación de un primer examen piloto en diciembre de 2001, con la participación voluntaria de $83 \%$ de los egresados de las Escuelas de Medicina de ASOFAMECH.

En mayo de 2002, se completó y aprobó la propuesta final por ASOFAMECH, y ésta se dirige a la sociedad, en especial a las instituciones vinculadas.

Se decidió hacer un ensayo final en diciembre de ese año, en el que participó el $100 \%$ de los egresados, experiencia que significó un positivo respaldo al diseño de ese examen y a los preparativos logísticos.

Los dos principales objetivos prácticos definidos del EMN son:

a) Aportar a la sociedad una forma objetiva de comparar conocimientos de los médicos que inician su ejercicio profesional en Chile.

b) Entregar información objetiva a las Escuelas de Medicina respecto a los conocimientos finales de sus egresados, con el fin de corregir eventuales falencias en áreas fundamentales.

Las principales características del EMN son que es un examen teórico, escrito, de opción múltiple, cuyo resultado es un puntaje en una escala continua de 1 a 100, dado por el porcentaje de 
respuestas correctas. No existe un puntaje mínimo de aprobación, por lo tanto, tampoco hay aprobados ni reprobados.

Algunas precisiones complementarias sobre sus características son que: en la forma en que está planteado, no es habilitante para el ejercicio de la profesión. Su uso tampoco está impuesto por ley, quedando a voluntad de los empleadores el exigirlo o no.

¿Por qué un examen teórico y no uno práctico? Idealmente, un examen con los objetivos que se plantean, debiera evaluar conocimientos, destrezas (intelectuales y técnicas) y actitudes de los médicos. En cuanto a la evaluación de actitudes, se ha considerado que debiera confiarse completamente a las escuelas formadoras, únicas con la oportunidad de observar al alumno en la gran variedad de situaciones que se requiere para ello.

El EMN sólo mide conocimientos y destrezas intelectuales del examinado. Otras destrezas no son susceptibles de medición en un examen de este tipo. Existen técnicas estandarizadas para evaluar destrezas técnicas y un buen número de actitudes, sin embargo, la experiencia nacional en estos sistemas de evaluación es incipiente, lo que sumado a la complejidad y costos de implementación, hacen recomendable postergar su uso.

a) El EMN mide: Nivel de conocimientos clínicos considerados comunes a todo médico que inicie su ejercicio profesional en Chile. Algunas destrezas intelectuales consideradas comunes a todo médico que inicie su ejercicio profesional en Chile.

b) El EMN no mide: Muchas de las destrezas que debe dominar un médico. Actitudes que debe poseer un médico. Conocimientos que no sean comunes a todas las Escuelas y que estén determinados por énfasis mayores en una Escuela respecto a otra.

¿Por qué un puntaje continuo y no simplemente aprobado/reprobado? El EMN entrega un puntaje dentro de una escala continua, expresada en porcentaje de respuestas correctas. El aprobado/ reprobado se descartó, ya que sólo tendría sentido si el examen fuese habilitante para el ejercicio de la profesión. Ambos objetivos del EMN exigen la necesidad de comparar entre egresados, lo que sólo puede lograrse con una escala continua.
No se trata de un examen habilitante para el ejercicio de la medicina. El EMN no hará diferencia en cuanto a la titulación ni el derecho a ejercer la medicina de ningún examinado. Las escuelas asociadas a ASOFAMECH han acordado voluntariamente exigir a sus egresados la rendición del EMN como requisito para la titulación, pero no se exigirá un puntaje mínimo de aprobación.

De manera que si bien sólo podrán titularse internos que se hayan "medido", el resultado de esa medición es irrelevante para la titulación.

¿Por qué del uso voluntario y no por "ley"? No se pretendía imponer por ley el examen, sino dejar a criterio de los empleadores el exigirlo para sus concursos, en la confianza de que la calidad y utilidad del EMN llevaría a éstos a utilizarlos progresivamente.

La obligatoriedad no es fácil de conseguir. Bajo la legalidad actual, un chileno en posesión de su título de Médico-Cirujano tiene el derecho a ejercer libremente la medicina de por vida. El título, a su vez, puede ser otorgado por cualquier universidad chilena con reconocimiento oficial.

De manera que no es posible agregar un examen obligatorio a los médicos ya titulados sin pasar por modificaciones legales (LOCE).

Los requisitos exigidos a un médico extranjero para ejercer en Chile, dependen de la existencia 0 no de convenios entre Chile y su país de origen, como ya se ha mostrado previamente.

No era posible agregar un examen nuevo a los médicos extranjeros sin modificaciones legales (incluida la denuncia (decisión de poner término a un convenio internacional) por parte de Chile de un convenio internacional).

Hacer obligatorio un examen para todos los médicos que comienzan a ejercer en Chile (esto es nuevos egresados y nuevos extranjeros), no era posible sin cambios importantes en la legislación vigente. Las gestiones políticas y los trámites legislativos que esto implicaría demorarían el inicio del proyecto y distraerían recursos.

Sin embargo, al margen de su dificultad, el hacer obligatorio el examen presentaría algunos inconvenientes que deberían tenerse presente:

a) La discusión de una ley generará debates interesados entre distintos actores del área de la salud (médicos extranjeros, gremios, nuevas universidades, Ministerio de Salud). 
b) Aun si se aprobara la ley, existe el riesgo de que la discusión legislativa distorsione el proyecto.

c) Al estar amparado en una ley, un examen se impondría aunque no fuese un instrumento útil o rigurosamente construido. Dejar su uso a criterio de los empleadores, en cambio, favorece en mejor forma su calidad y utilidad.

d) Un instrumento cuyo uso se base en la aceptación y necesidad pública es más estable que uno impuesto por ley. Puede demorar más en establecerse, pero lo hace de manera más sólida. La obligatoriedad legal puede parecer atractiva como un camino de implementación general más rápida, pero se le puede poner fin en forma igualmente expedita.

Contenido del examen. Los contenidos del examen serán aquellos comunes a los currículos de las escuelas de ASOFAMECH.

Se descartó el tratar de confeccionar el perfil de conocimientos "del médico que Chile necesita", por la imposibilidad de definir este médico hipotético, que hoy se desenvuelve en los más diversos ambientes, con grandes diferencias en la disponibilidad de recursos, prevalencia de patología, posibilidad de derivación, etc. Se descartó, asimismo, el llegar a definir "los conocimientos mínimos que todo médico debe poseer", también difícil de definir, puesto que la situación de cada médico le exige dominar un subconjunto de esos conocimientos.

Se optó por un camino más práctico: definir un perfil de conocimientos comunes a todas las escuelas. Lo que se persigue es describir en forma específica cada uno de los temas que son enseñados en todas las escuelas, así como el nivel de profundidad del conocimiento en cada uno de ellos.

La ventaja de esta aproximación es que la lista de conocimientos generada será de consenso y, por tanto, no cuestionada; la confianza que esto generará, redundará en la viabilidad del proyecto y su aceptación por la comunidad.

La matriz del EMN. Los objetivos del EMN exigen que los puntajes de dos médicos sean comparables aunque hayan rendido el examen en años distintos, tanto para competir por una posición, como para que una escuela pueda comparar una generación con otra, sin embargo, los conocimientos que se deben exigir a un médico van cambiando con el tiempo. Los avances científicos pueden hacer surgir nuevas áreas de la medicina, así como dejar otras obsoletas; el cambio de la situación epidemiológica del país obligan a prestar más atención a ciertas áreas que a otras. Estas dos necesidades, por una parte mantener un examen lo más comparable posible en el tiempo y, por otra, ajustarlo a los tiempos, deben ser recogidas en el diseño de la matriz del examen.

En la primera versión oficial del EMN el elemento central de la matriz del examen lo constituyó "el peso relativo" de cada una de las siete especialidades, o áreas del conocimiento médico, en el universo de preguntas del EMN, expresado en términos porcentuales.

Después de un detenido análisis, se consensuó entre las diferentes Escuelas de ASOFAMECH la siguiente distribución porcentual de las preguntas en las diferentes especialidades, porcentaje que a su vez refleja el "peso real" que tienen en los programas de estudio actuales de las diferentes Escuelas de Medicina las referidas especialidades o áreas del conocimiento.

$\begin{array}{lr}\text { Medicina Interna: } & 37 \% \\ \text { Cirugía: } & 12 \% \\ \text { Pediatría: } & 16 \% \\ \text { Obstetricia y Ginecología: } & 16 \% \\ \text { Psiquiatría: } & 8 \% \\ \text { Administración en Salud: } & 5 \% \\ \text { Especialidades: } & 6 \%\end{array}$

El grado de dificultad de las preguntas no fue incorporado a la matriz, ya que idealmente, de acuerdo a los objetivos del examen, todas las preguntas deberían tener una dificultad mediana para que la curva de resultados globales tenga una distribución normal.

En el futuro podría considerarse otros elementos en la matriz.

Producción del examen. La producción del examen comienza con la solicitud de preguntas a las Escuelas de Medicina pertenecientes a ASOFAME$\mathrm{CH}$. Esta solicitud es realizada por el director del Examen Médico Nacional a los directores de cada Escuela. En la solicitud se especifica el número de preguntas requeridas y los plazos para entregarlas, pero no el mecanismo para crearlas, quedando el 


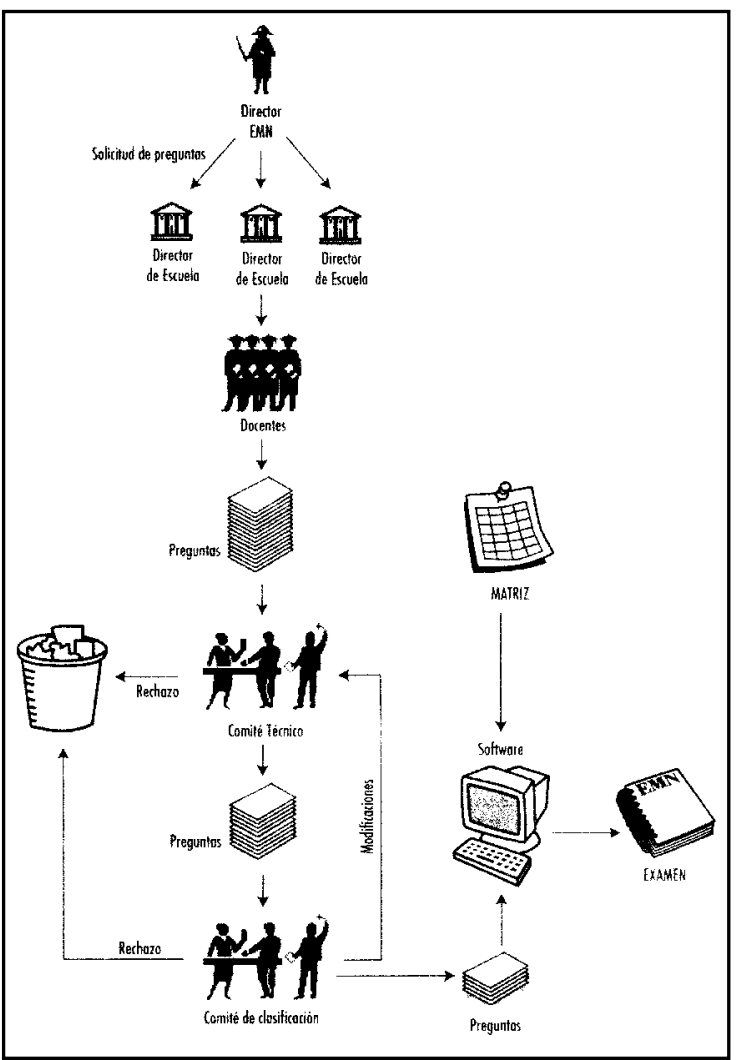

Figura 1. Flujo de producción del Examen Médico Nacional.

director de la Escuela en libertad de acción en este sentido. La solicitud también menciona las especialidades que considera el examen, la presencia relativa de preguntas para cada una de ellas, y cualquier otra información relevante.

Estandarización de las preguntas. La estandarización estará en manos del Comité Técnico del EMN, compuesto por educadores y médicos competentes en la materia. Este grupo se reunirá periódicamente para revisar cada pregunta recibida. La revisión tiene dos objetivos: estandarizar el lenguaje y asegurar una correcta estructura técnica y lógica de las preguntas.

Revisión de contenidos y clasificación de las preguntas. Las preguntas aprobadas por el Comité Técnico serán luego revisadas por el Comité de Clasificación. Los objetivos de esta etapa son: a) Asegurar que las preguntas correspondan a conceptos sobre los cuales exista consenso médico y que, además, correspondan al perfil de conocimientos comunes de los egresados chilenos mencionado más arriba.

b) Clasificar cada pregunta de acuerdo a sus propiedades, tales como la especialidad a que corresponde, su grado de dificultad, tiempo esperado de vigencia, etc. Esta clasificación es indispensable para utilizar la matriz mencionada y para administrar el banco de preguntas.

El Comité de Clasificación se reunirá periódicamente, en sesiones dedicadas a preguntas de una misma especialidad, asesorándose en cada sesión por un grupo de especialistas del área (un especialista por Escuela).

El Comité podrá aprobar o rechazar una pregunta, pero no podrá realizar modificaciones. Para comegir algún aspecto del contenido, la pregunta deberá reenviarse al Comité Técnico con la sugerencia comespondiente. Esta prevención es necesaria, ya que una modificación de contenido puede repercutir en la estructura formal o lógica de la pregunta.

Las decisiones de este Comité deberán tomarse por unanimidad, ya que los contenidos de las preguntas deben ser de consenso entre las Escuelas.

Las preguntas aprobadas por el Comité de Clasificación conformarán el bando de preguntas en carácter de definitivas o clasificadas.

En cuanto al lenguaje, éste debe ser claro, sin ambigüedades ni localismos (español internacional). La estructura de las preguntas y su sintaxis debe ser común para todas. El vocabulario médico que se utilice debe ser estándar y el mismo en todas las preguntas.

Para lograr estos objetivos, el Comité podrá modificar las preguntas en cualquiera de sus aspectos formales, pero no en aspectos de contenido. Si la corrección de una pregunta exige modificaciones de fondo, ésta será eliminada del banco de preguntas. Las preguntas aprobadas, tomarán categoría de estandarizadas.

Uso y publicación de los resultados. Existen 4 potenciales interesados en acceder a los resultados del examen: el examinado, las escuelas, los empleadores y el público.

Se ha estimado que el acceso del público general a los resultados individuales de los egresa- 
dos generaría distorsiones indeseadas, ya que se corre el peligro de transformarlos en una forma de comparación muy limitada entre profesionales, que no consideraría aspectos fundamentales de la calidad del médico, ni su experiencia posterior al egreso.

$\mathrm{Si}$ bien los empleadores serán beneficiarios directos del examen, que les entregará un elemento objetivo de comparación entre postulantes, se ha estimado que el acceso a la totalidad de los resultados podría generar distorsiones en la interpretación de éstos, corriéndose el riesgo de que se tienda a descalificar, infundadamente, escuelas completas, siendo la intención del examen la comparación entre individuos. Al empleador sólo debieran interesarlo los resultados de los individuos que postulen a su institución.

Por estas razones, se ha determinado que se entregarán los resultados solamente al examinado y a las escuelas participantes.

Información al examinado. El examinado recibirá una tarjeta oficial con sus resultados y será de su total arbitrio el utilizarla. El empleador podrá exigir el resultado como requisito de postulación a algún cargo o cupo en programas de especialización, pero sólo podrá acceder a él a través del postulante.

La información al postulante incluirá su puntaje promedio global.

Información a las Escuelas de Medicina. Cada escuela recibirá la información completa para cada uno de sus egresados, esto es puntaje general y puntaje por área para cada examinado. Además de este reporte, cada Escuela de ASOFA$\mathrm{MECH}$, recibirá los resultados agrupados para cada una de las otras Escuelas, sin especificar individuos, incluyendo los resultados generales. No se entregarán resultados pregunta por pregunta, los que serán de uso exclusivo del EMN. Esto último para evitar tanto la filtración de las preguntas, como el intento de corregir infundadamente aspectos demasiados específicos de los contenidos curriculares por parte de las Escuelas.

Uso publicitario de los resultados. Las universidades participantes suscribieron un acuerdo por medio del cual se comprometieron a no hacer uso de los resultados del EMN en ningún medio público ni con fines publicitarios. Sólo podrán dar información pública del examen ASOFAMECH.

Posibles efectos adversos del Examen Médico Nacional. Es indudable que la introducción de un examen de este tipo significa un cambio importante, tanto en la educación médica, como en las políticas de selección en concursos de graduados y selección profesional. Las Escuelas de Medicina agrupadas en ASOFAMECH están convencidas que estos cambios son necesarios y que los beneficios derivados de su introducción superarán con creces a sus eventuales efectos negativos.

Las comisiones que han diseñado el presente examen han intentado, desde el comienzo, considerar las posibles consecuencias no deseadas del EMN. Para ello se realizaron consultas amplias a los actores más importantes en el tema. El diseño propuesto para el EMN aborda gran parte de las observaciones iniciales.

Los más importantes reparos recogidos son:

a) Al medir sólo aspectos cognoscitivos, el examen dejanía de lado importantes aspectos de la calidad de un médico, tales como la relación médicopaciente, el espíritu de servicio, valores éticos, pericia diagnóstica y terapéutica, etc. Acentuando de paso el negativo carácter "memorístico" que ya poseen los estudiantes de Medicina.

b) El éxito de una Escuela estaría dado por su puntaje en el examen. Esto favorecería a las Escuelas con débil componente práctico (laboratorios, campos clínicos). La presión sobre las Escuelas de Medicina para reforzar los temas incluidos en el examen las obligaría a disminuir el tiempo dedicado a ramos básicos, formación general y enseñanza práctica. Esto conllevará también que el currículo se verá manejado "desde fuera", rigidizándose, con la consiguiente pérdida del carácter distintivo de las Escuelas, dificultando de paso las experiencias renovadoras.

c) El examen también afectaría directamente a los alumnos, ya que la preparación del examen significará gran presión sobre ellos. Posiblemente surjan, como en España, academias de preparación del examen, en desmedro de los alumnos con menos recursos.

El EMN mide fundamentalmente los conocimientos del examinado, la integración de los 


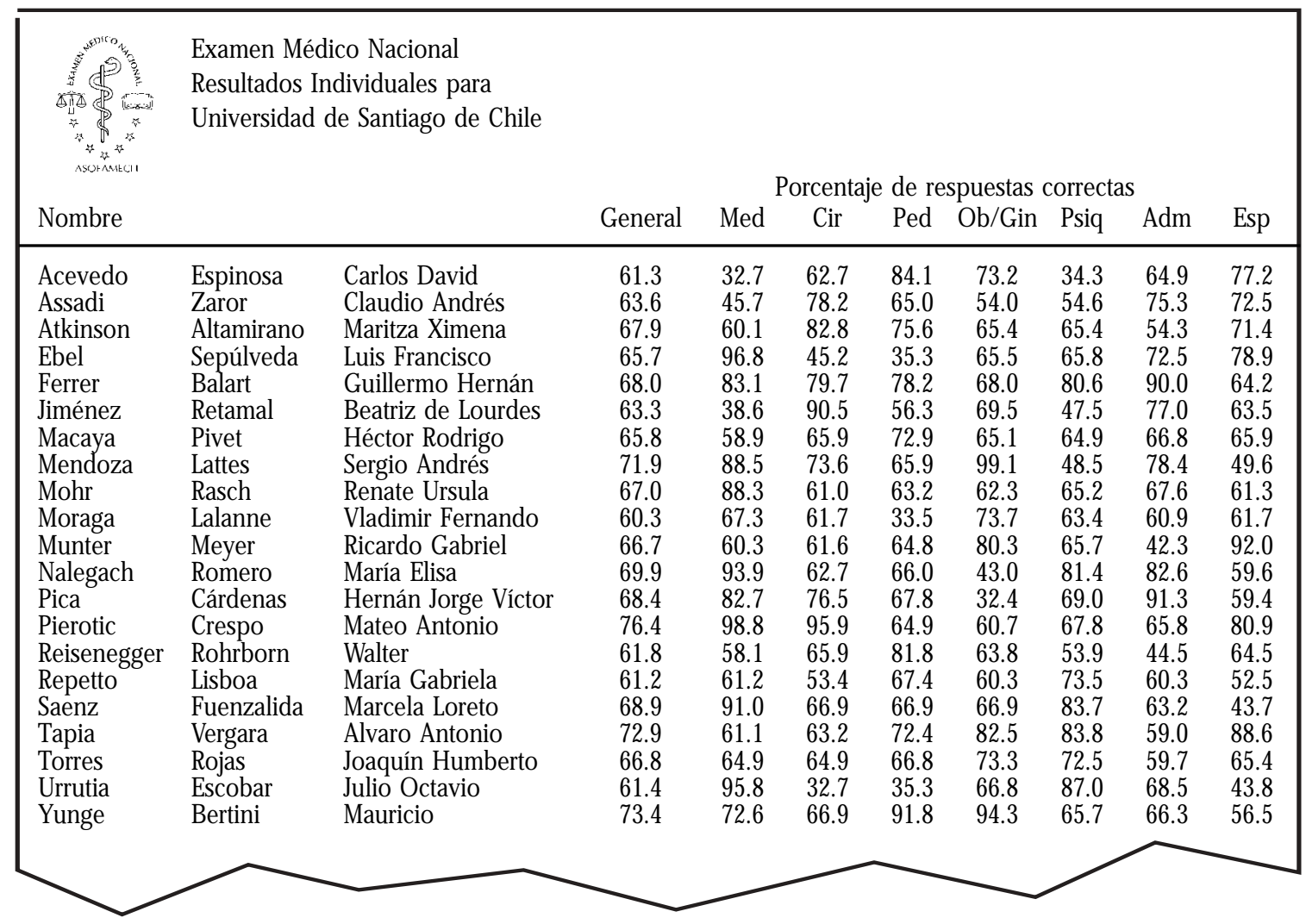

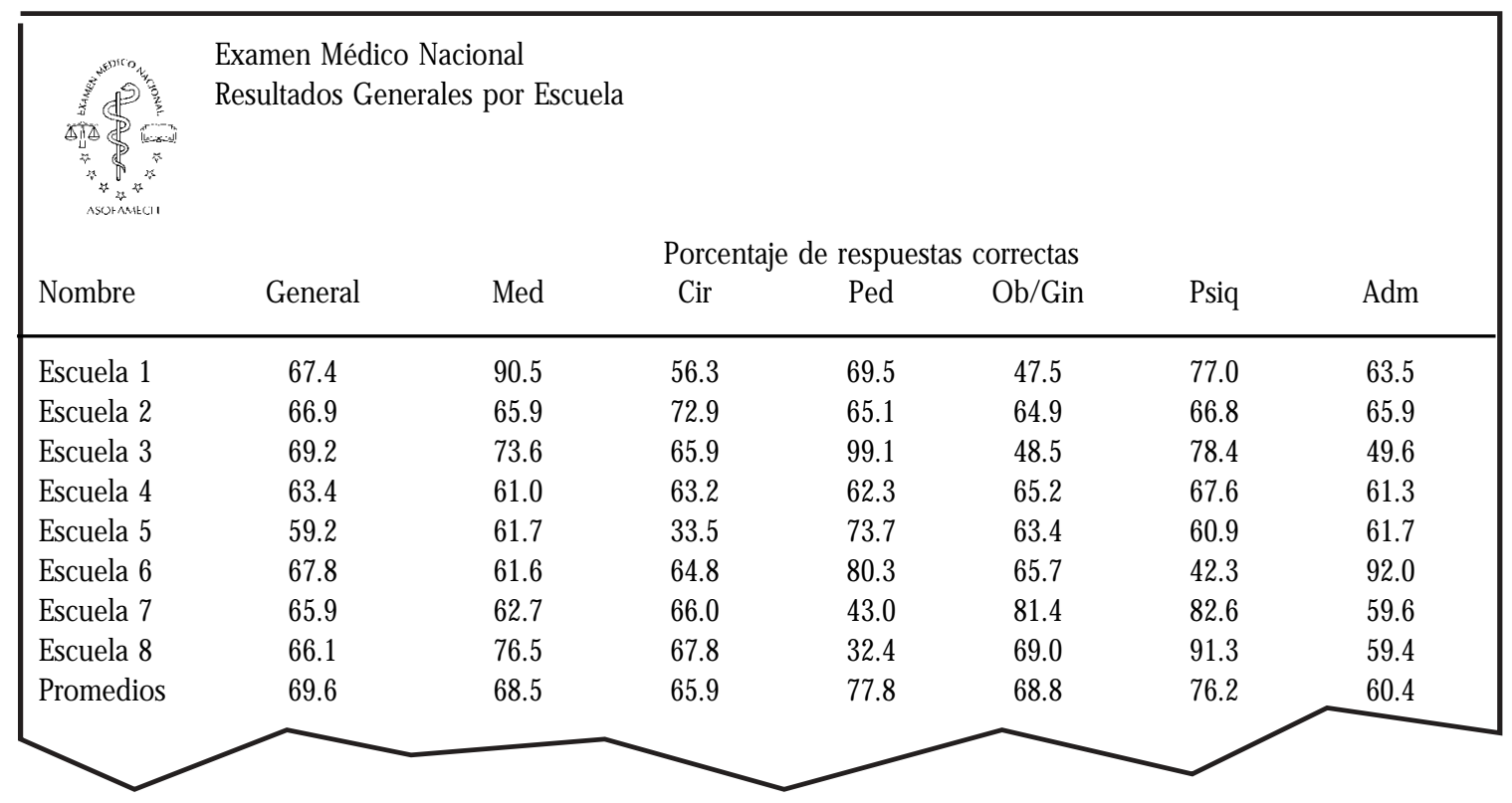

Figura 2. Estas figuras son ejemplos simulados para informes de resultados por Escuelas. 
mismos y algunas destrezas intelectuales. Las destrezas que hoy no son susceptibles de medición podrán serlo a futuro, en la medida que se implementen sistemas de evaluación práctica estandarizada. En cuanto a los aspectos valóricos de la formación médica, se trata de características difíciles de medir, cualquiera sea el método empleado. Velar por estos aspectos queda confiado a las respectivas escuelas, que tienen oportunidad de conocer a sus alumnos a lo largo de muchos años y expuestos a situaciones muy diversas. Para informarse de estas actitudes, los eventuales empleadores cuentan con otros elementos: prestigio de la institución de donde proviene, recomendaciones, entrevistas personales, ensayos, evaluaciones psicológicas, etc., todos ellos procedimientos de uso común.

Con el fin de no promover hábitos de aprendizaje "memorísticos", será función prioritaria de los comités técnicos responsables de la producción del examen, descartar las preguntas orientadas a la memorización de datos, privilegiando aquellas orientadas a la integración de conocimientos, aplicación práctica y capacidad analítica.

En cuanto a las eventuales distorsiones a nivel del currículo de las escuelas, éstas se verán minimizadas desde el momento en que las universidades participantes firmarán un acuerdo en el que se comprometerán a no hacer uso publicitario ni público de ninguno de los resultados del examen. Los resultados globales por escuela, serán de uso interno y persiguen reforzar áreas débiles. Si se considera que el EMN sólo cubre contenidos mínimos, comunes a todas las escuelas, se comprende que de ningún modo es inadecuado que las escuelas corrijan eventuales deficiencias en esas áreas, para lo cual se debe confiar en su madurez, autonomía y buen juicio.

La estandarización de los contenidos elementales del currículo será sin duda una de las consecuencias del EMN, lo que no es visto como un efecto negativo. No olvidemos que estamos hablando de contenidos comunes en los que hay consenso entre muy distintas escuelas. Queda un amplio espacio para todos los contenidos adicionales que confieren el carácter y estilo a cada escuela.

Respecto a las consecuencias negativas sobre el alumno, hay que aclarar que el carácter del examen no es tan decisivo, ya que no es habilitante, ni tan extenso, puesto que sólo evalúa contenidos clínicos, de manejo reciente por el alumno en internados, sólo se contemplan conocimientos básicos cuando tienen directa relevancia clínica. Los contenidos del examen están basados en los contenidos curriculares de los ramos clínicos de las carreras de medicina existentes, de manera que el mejor lugar para preparar el examen es simplemente la Escuela de Medicina.

Cierto nivel de angustia es inherente a cualquier examen útil, y difícil de evitar; sin embargo, se procurará disminuir este efecto poniendo a disposición de los examinados material en forma de facsímiles, perfiles de conocimientos por especialidad, la matriz con el peso relativo de los temas, etc., con el fin de minimizar el estrés que la ausencia de información pueda generar.

ASOFAMECH está convencida de la necesidad de seguir avanzando en el control de calidad de la formación y el ejercicio médico en el país y el EMN es un paso importante en esa dirección. Provocará cambios beneficiosos, pero los efectos adversos discutidos en este capítulo deben ser tenidos en cuenta, y las propuestas planteadas para evitarlos deben estar sujetas a permanente revisión y perfeccionamiento.

\section{ASPECTOS REGLAMENTARIOS}

Quienes rinden el examen. Todos los egresados de escuelas afiliadas a ASOFAMECH deberán rendir el examen, como requisito obligatorio para titularse. Cualquier escuela no afiliada podrá inscribirse para que sus alumnos lo rindan, esto no las obliga a exigirlo obligatoriamente a sus egresados.

Podrá rendirlo en forma individual y voluntaria cualquier egresado de escuelas no afiliadas, así como cualquier médico, sea chileno o extranjero, estos últimos a partir de 2005.

Cuándo se rinde el examen. Por tratarse de un requisito para el título, esto debe ocurrir antes de la titulación, y por evaluar el total de los conocimientos clínicos de los egresados, debe ocurrir luego de terminar éstos su formación, lo que deja una breve ventana abierta entre el egreso y la titulación. Esto requiere un esfuerzo de coordinación de las actividades de fin de año por parte de las Escuelas participantes. 
Dónde se rinde el examen. En principio, el examen se rendirá en cada una de las Escuelas de Medicina inscritas, incluyendo a aquellas no afiliadas a ASOFAMECH.

Los inscritos a título personal deberán inscribirse en la sede de su conveniencia.

Oportunidades para rendirlo. Un individuo que desee mejorar su puntaje, podrá rendirlo hasta tres veces. Se estima por parte del Comité Directivo del EMN un plazo máximo de tres años desde el egreso para rendir nuevamente el examen.

Para estos casos sólo aparecerá un puntaje en la tarjeta de resultados: el de mayor nivel.

Situación de los egresados anteriores al EMN. Durante los primeros años de entrada en vigencia del EMN, se dará una situación en que competirán por cargos y becas, médicos que no rindieron el EMN con aquellos que sí lo hicieron. La única solución práctica a este problema, es dejar a los empleadores decidir los criterios para comparar a unos y otros. Nunca se ha planteado el EMN como un criterio único de selección, sino como un antecedente objetivo de comparación de conocimientos.

Los empleadores son los únicos que pueden establecer criterios de equivalencia para comparar - por ejemplo- a un médico con dos años de experiencia práctica con uno sin experiencia pero con un excelente puntaje.

Por último, si un empleador decide exigir el EMN como requisito obligatorio de postulación, el postulante siempre tiene la posibilidad de rendir el examen.

Funcionamiento del EMN. El EMN cuenta con una sede equipada, independiente de cualquiera de las universidades participantes. Esta sede estará ubicada en Santiago y contará con un personal mínimo. El grueso de su función estará dada por una importante participación de académicos de todas las escuelas en los comités.

El EMN está a cargo de un Director Ejecutivo, nombrado por ASOFAMECH, este Director tiene amplias atribuciones, con el fin de agilizar al máximo la puesta en marcha del proceso. El Director debe rendir cuenta a un Comité Directivo, conformado por un representante de cada Escuela. Esta cuenta ocure dos veces al año, en fechas predefinidas.
Se han definido con precisión ciertas decisiones que el Director no podrá tomar sin la autorización del Comité Directivo, fundamentalmente en lo que compete a la difusión pública de resultados y a la modificación de estatutos y reglamentos. Para estos casos deberá consultar al comité en forma extraordinaria.

Examen Médico Nacional 2003. La primera versión oficial del EMN en diciembre de 2003, significó la participación de todos los egresados de las Escuelas de Medicina de Chile. En total 760 médicos, provenientes de ocho Escuelas de Medicina pertenecientes a ASOFAMECH a la fecha (U. de Chile, PU Católica de Chile, U. de Concepción, U. de Valparaíso, U. de la Frontera, U. Austral de Chile, U. de Santiago y U. de Los Andes) y de tres Escuelas no asociadas (U. Antofagasta, U. Católica de la Santísima Concepción y U. San Sebastián). La totalidad de las Escuelas de Postgrado de las Facultades de Medicina de ASOFAMECH exigieron, a partir de 2004, como requisito para postular a una plaza de especialización el haber rendido el EMN. El Ministerio de Salud, por su parte, ha planteado formalmente su decisión de establecer, a partir del próximo año, como requisito para contratar a un médico en cargos públicos el haber rendido el EMN.

\section{ESPECIALIZACIÓN MÉDICA DE POSTÍTULO}

En los últimos treinta años, se ha consolidado en Chile como uno de los componentes fundamentales de la formación médica la especialización de postítulo a través de programas formales, en general de tres años para las especialidades primarias y de dos años adicionales para las llamadas especialidades secundarias o derivadas. Son programas de dedicación plena y de alta exigencia tanto en los aspectos cognitivos como de habilidades y destrezas y actitudes. Esta especialización de la medicina en el marco de un desarrollo científico y tecnológico de crecimiento exponencial en un signo relevante de los tiempos presentes y del futuro para la educación médica. ASOFAMECH en forma pionera en Chile y ciertamente visionaria, estableció en 1979 un sistema de acreditación de los diferentes programas y centros formadores de especialistas de su dependencia. A 
través de siete ciclos sucesivos de acreditación se han ido efectuando ajustes, correcciones y mejoras en la formulación y desarrollo de los diferentes programas y de los centros docentes asistenciales donde se desarrollan para asegurar la calidad de los mismos y por ende las competencias de los médicos especialistas en un creciente y diverso universo de especialidades médicas. Considerando esta experiencia y por la gran pertinencia social, calidad y exigencias de la formación de especialistas en medicina, ASOFAMECH, solicitó al ejecutivo la incorporación de la acreditación de los programas de formación de especialistas en medicina al proyecto de ley de aseguramiento de la calidad de la educación superior, lo que fue acogido.

Por otra parte, en 1984, ASOFAMECH, participa junto a las Sociedades Científicas Médicas, al Colegio Médico y a representantes del Ministerio de Salud en la creación de una Corporación Nacional Autónoma para evaluar y certificar la condición de especialistas (CONACEM) de los médicos que buscaban ese reconocimiento por parte de sus pares y de la sociedad toda. Para acceder a esta certificación, CONACEM establece principalmente la exigencia de un título universitario de un programa acreditado o la aprobación de un examen teórico y práctico ante una comisión especial, previa verificación de una experiencia mínima de cinco años en un servicio clínico calificado.

En el marco de la reforma de la salud la nueva ley de Autoridad Sanitaria, crea una Superintendencia de Salud donde quedará radicado un registro nacional de médicos especialistas. Al respecto, ASOFAMECH, ha planteado a las Autoridades Ministeriales y a la sociedad toda, que el acceso a este registro y por ende al reconocimiento oficial de la condición de especialista, debe estar vinculado directamente a un título universitario de un programa de especialización acreditado 0 a una certificación de CONACEM.

Estos son los procesos y mecanismos que ASOFAMECH ha desarrollado para asegurar la calidad de educación médica de pre y postgrado en Chile y que a su vez representan los instrumentos que permitirían reconocer las condiciones para la confianza en la movilidad de profesionales extranjeros hacia nuestro país para el ejercicio de la medicina. 